\title{
The Design of Reference Service System in Cordova-based Hybrid Frameworks
}

\author{
Dengke XIN, Lei ZHANG*, Xiaoliang YANG, Yuan FANG
}

\begin{abstract}
With the rise of mobile technology, the library reference service has dramatically changed. Targeting the new requirements, this paper aims to design a new library reference service system in Cordova-based hybrid frameworks, which caters to the web service embedded in two major mobile platforms, iOS and Android, as well as the PC platform. The new system adopts the WebSocket based technology to realize the function of independent online reference, which improves the quality of the normal digital reference service. The newly designed system also applies the ECS cloud server technology, thereby significantly slashing the hardware setup cost, extending the basic reference service, and improving its fitness-for-use and convenience, and optimizing the allocation of local resources.
\end{abstract}

Keywords: Cordova; DRS; ECS cloud technology; Hybrid Frameworks; WebSocket

\section{INTRODUCTION}

Digital Reference System (DRS) inherits, extends, and further develops the characteristics of traditional reference systems in the internet era [1-3]. As a new type of information service mode, it integrates the Digital Library System, the Library Information Service Mechanism, and the Service Mechanism for the user. It is a key indicator that reflects the capability and quality of library service. In the early 1980s, the book and information sectors in Europe and the United States designed DRS [4-6]. After two decades of development, DRS witnessed dramatic progress and was gradually improved upon, with the U.S. as the typical representative. As a matter of fact, DRS originated from the U.S., in which the health and science library of Washington University and the health service library of Barry University together launched the "Electronic Reference System", marking the first DRS that operated online. In 1999, the Library of Congress in the U.S. started to initiate the Collaborative Digital Reference Service (CDRS), which was expected to become a digital reference service item for international cooperation. The aim of the CDRS was to provide professional reference service to anyone who came up with any question, at any time, and at any place.

In June 2002, CDRS developed into QuestionPoint, a cooperative reference website jointly created by the Library of the UN and the Online Computer Library Center (OCLC). Users can take advantage of the QuestionPoint online reference service by clicking into the ASK webpage. Similarly, VRD technology also adopts such an idea. It is a cooperative consultancy project funded by the U.S. Department of Education, the virtual reference desk of which is composed of a distribution Meta Triage system and several ASK websites. Users have access to the system via the web, and raise questions via e-mails or online consultancy. Its Meta Triage system then classifies and handles those questions [7-8]. According to the statistics, of all 122 libraries affiliated with Association of Research Libraries (ARL), 96\% have already launched DRS in different forms [9-10]. At present, the mobile reference service in China is still in an early development stage in terms of theory and practice, but the content of conventional reference service, and the way to provide it, is relatively simple. The mainstream reference services are still primarily dependent upon traditional in-campus platforms, e-mails, short message service (SMS), or a combined reference service platform that integrates a few or all the traditional platforms. However, the compatibility of communication among these platforms needs to be improved, and the cost for development is relatively high [11]. The mobile terminal-based reference service emerged only in recent years. China faces many challenges in the development of Reference Service System, mainly as follows:

a) The mobile reference service develops extremely uneven.

The progress of reference services in various regional libraries is quite different. Although in some economically robust and technologically advanced regions, the mobile reference services are already put into use, quite a lot of college libraries in these regions lack a complete system framework despite their digital reference services. Even worse, no reference service is ever established in the backward areas. How the local library reference service system develops is closely related with the local economy and the need of local institutions and universities.

b) The current mobile reference systems have problems such as oversimplified service mode and insufficient personalized service.

The Mobile information services in China may copy the patterns of Western countries, leading to an oversimplified development mode. Under such mode, most of them seek to cooperate with the third-party for technical support, thereby cutting their own development costs on technology and finance, but this cooperation mode lacks flexibility due to the technical limitations of the third parties and may lead to incompatibility between the systems of the libraries and the third parties.

c) Limited $R \& D$ technology for mobile reference service and incompatibility of multi-platforms for system R\&D.

Due to insufficient strength in mobile service technology in local libraries, and despite the staff of the library being familiar with the content and process of the current mobile information service, they are still incapacitated for R\&D and maintenance. As the library reference service now is in a transfer stage from the digital reference service to the mobile reference service, it cannot completely abandon users who are already familiar with 
computer based operation. Therefore, the library information service system also needs to be compatible with PC platform.

d) Insufficient R\&D fund for mobile reference service in libraries.

As the fund pool for colleges and universities is limited, and the schools have to invest heavily on the necessary projects such as personnel training, discipline optimization and capacity building for faculty to improve the level of education, the libraries actually gained very limited funds each year. Besides, apart from the necessary plunge in infrastructure upgrades and maintenance, the money used for R\&D and building of library service system platforms which usually requires higher cost is meager. Actually, insufficient funds are a common problem faced by most libraries. Only a few colleges and universities have enough money to invest in the R\&D of similar service system. Therefore, this caused certain restrictions on the promotion and localization of mobile book information service.

In order to sort out the above-mentioned problems, the paper designs a reference service system that is compatible with various platforms, which is based on the hybrid platform development model and adopts Cordova and ECS cloud technology. The system takes advantage of Cordova technology to cater to both the PC platform and the Application Program Interface (API) on mobile operating systems like Android and iOS. This in turn allows for software development in the hybrid frameworks. The system is based upon WebSocket technique, which leverages the common function modules and provides realtime reference service independently, and has greatly slashed the cost for development. Therefore, this system can better meet the requirements for service extension in local university libraries, while offering a more convenient and faster platform for its users.

\section{THE HYBRID PLATFORM BASED REFERENCE SYSTEM}

Compared with developed countries, China's reference service started late and witnessed uneven development among different regions. For backward areas, it even needs to face the heavy cost on R\&D and facilities building. In response to user needs, the local libraries in China have to think about how to provide the best quality reference services under current conditions. The paper intends to solve the above-mentioned issues by using a hybrid platform based reference system.

\subsection{Connotation and Characteristics of the Hybrid Platform Based Reference Service}

With the rapid development of network technology and mobile communication technology, a new stage of reference service model is gradually emerging. In view of the problems regarding the promotion and setup of local libraries, and to maintain the advantages of reference services in the current stage and to expand the demand for new mobile reference services, the hybrid platform reference service gradually comes into being. By virtue of the compatibility mode technology of the hybrid platform, the specific service content includes not only providing web related services on $\mathrm{PC}$, but also the web related service on the mobile terminal. It is a product consisting of both digital reference service and mobile reference service, with the latter as the main characteristic. It not only combines the advantages of both, but also weakens the shortcomings of both. Although there are some technical immaturities, it can provide users with quality services.

The hybrid platform based reference service system designed in this paper has strong fitness-for-use, practicability and ease of use. It not only inherits and combines the advantages of various types of reference consulting services, but also has its own characteristics.

a) The Hybrid platform reference system has lower development cost, stronger compatibility and better cost/performance.

With the development of compatible platform technology that integrates both network technology and mobile communication technology, it has obtained a solid technical foundation for the hybrid platform technology and a more matured reference module theory, thus greatly reducing the development cycle. The hybrid system simultaneously is compatible with $\mathrm{PC}$ and mobile communication devices. It matches well the two major mobile phone systems (Android and iOs). Combining the advantages of digital reference system that is based upon computer network and the advantages of mobile reference system that is based upon mobile communication technology, it fully leverages the information science and technology of modern days. Besides, this hybrid platform based reference system will streamline the local server configuration by adopting an online server and provide flexible and dynamic online configuration by keeping abreast of the system busyness, thereby cutting unnecessary server costs.

b) The hybrid platform based reference service system targets more customer groups and its environment is more open and diversified.

As the new system combines two reference services, so its service environment and groups are more diversified. At the same time, the system not only retains user groups of digital reference service, but also expands its service to user groups of mobile reference service. So, it has more user groups. Besides, it can learn more about its user needs through synchronous or asynchronous interaction, thus providing services that are more suitable for individual needs.

c) The hybrid platform based reference system provides a more convenient, fast and stable service.

The platform provides both PC-fixed network service and mobile network service to ensure that the service can be accessed to by users on a PC fixed network, or by users on mobile networks. In this way, it provides more convenient and fast service to users with different needs. So, the two services are mutually alternative and mutually supportive to each other, thus providing users with a more stable service. The hybrid platform based reference service system not only cuts the development costs, but also streamlines requirements for server setup. The paper aims to use the hybrid platform technology to solve the common problems regarding the reference service among local universities in China. To be specific, The Cordova hybrid platform technology is used to set up a software platform that is adaptive well to both mobile terminals (with iOs system and Android system) and PC system. The system 
adopts Web Socket's two-way communication technology to set up an integrated platform allowing multi-person and real time online service. Besides, by combining with the flexible ECS cloud storage technology, it will significantly slash the hardware cost of the server [12].

\subsection{Technologies for Hybrid Platform Based Applications}

a) Cordova hybrid platform technology.

At present, the mainstream mobile platforms such as Android and iOS in the mobile communication technology are developed separately, and their fundamental technologies are totally different. Therefore, it forced the software companies to develop applications separately for different platforms. Moreover, it will result in fragmentation effects when developed separately. Cordova technique is a mobile development framework that serves as a middle connection between the local mobile terminal API and internet applications [13].

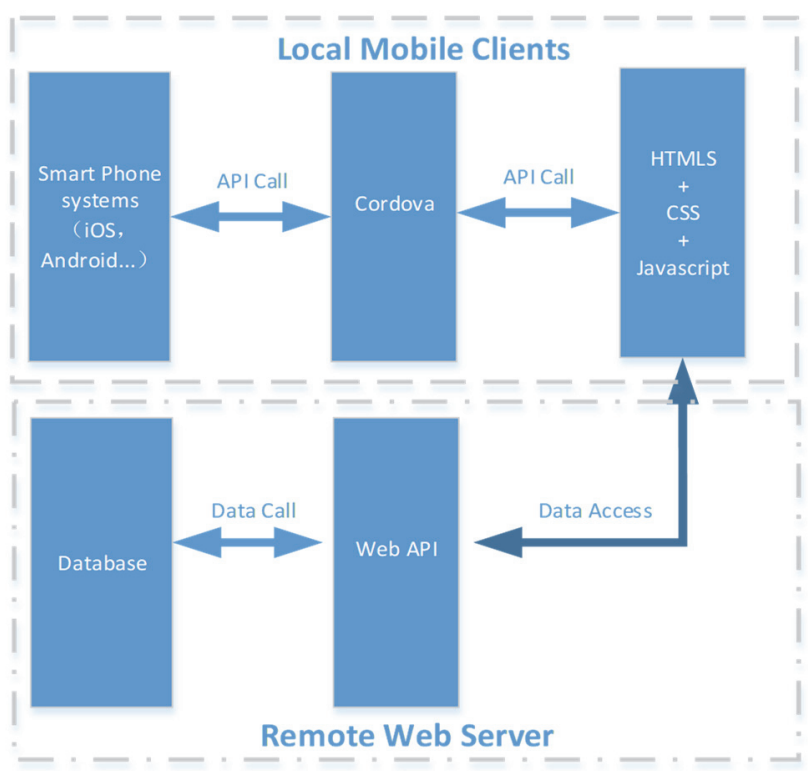

Figure 1 APP structure based upon the classical Cordova framework

As Fig. 1 indicates, for the extension and package of WebKits affiliated to different smart systems, Cordova is able to call JavaScript through WebView to establish communication between the terminal server and several clients. Additionally, Cordova has a library containing many different plug-ins, and its object-oriented development mode resolves the problem of needing to adapt to the API of different cell phones. The system front webpage, which is based upon UI framework and assemblies, can adapt to multiple devices, different monitor sizes, and different display resolutions. Furthermore, the Cordova framework also provides an API that allows JavaScript to access the mobile platform, and supports mainstream mobile development platforms (iOS, Android, Windows XP, and so forth). The low level of the mobile terminal adopts the plug-ins and API terminals of Cordova, and adapts to diverse mobile devices. This provides the system service with a good package, and allows for repeatability and easy extension and maintenance. This, in turn, establishes a foundation for the system to realize a cross-platform feature [14]. The application created by Cordova is still packaged as an SDK application, and can be downloaded and installed easily. The cloud package app of Cordova is compatible with all the operating systems, including iOS, Android, Windows phone, Blackberry, and Symbian [15]. An application structure based upon the classical Cordova framework is shown in Fig. 1.

Referring to the design requirements of consultancy R\&D system and basing upon Cordova cross-platform technology, the paper develops a system that is well compatible with PC system and mobile terminal systems like iOS and Android operating systems.

b) Web Socket's two-way communication technology

Web Socket is a TCP protocol that allows for fullduplex communication between browsers and servers. It was first proposed in 2008 and set as an international standard by the Internet Engineering Task Force in 2011 (IETF). The Web Socket protocol is applicable to all present-day browsers. The biggest advantage is that the server can actively push information to the client, and the client can also actively send messages to the server, thereby establishing a two-way equal and instant dialogue as shown in Fig. 2.

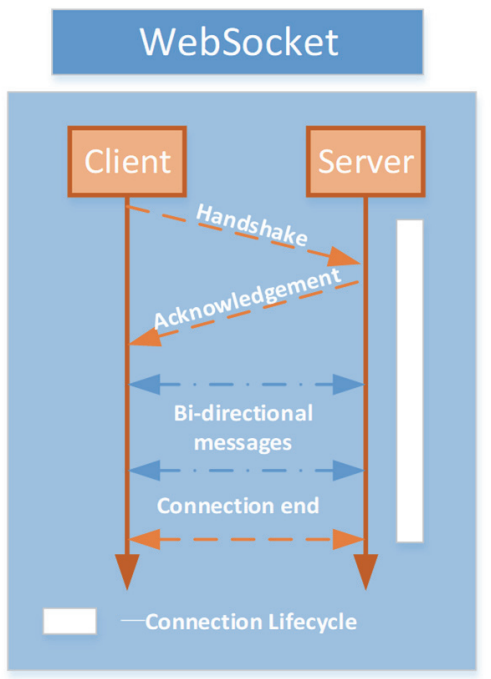

Figure 2 Web Socket protocol's two-way equal dialogues

Basing upon the Web Socket protocol, the paper further builds a two-way online consulting platform that can make full use of the compatibility between Web Socket protocol and HTTP protocol, and combine with the Web technology of the hybrid platform based reference system, enabling PC and mobile terminal users to obtain similar reference service.

c) ECS technology of Cloud server.

The Elastic Compute Service (ECS) is a cloud computing virtualization service provided by Alibaba Cloud. It is based upon the addition, use and delivery mode of Internet-related network servers. Before use, the clients do not need to purchase hardware devices in advance and they can create relevant servers on the network cloud at any time in accordance with their own business requirements by configuring specific parameters and selecting the desired ECS cloud servers. When using, users can expand the services by enlarging the disk volume and increasing the bandwidth of the cloud server at any time; if users do not need the cloud server, they can release resources at any 
time and save the cost.

The paper, by adopting the ECS technology, streamlines the cumbersome work of building a local physical server system and cuts its high cost, thereby significantly slashing the R\&D cost of the hybrid reference platform and making it possible to provide and popularize the reference service with good performance/cost to local university libraries.

\section{THE DESIGN OF REFERENCE SERVICE SYSTEM (RSS) IN HYBRID FRAMEWORKS}

\subsection{Objectives of Reference Service System (RSS)}

This paper aims to design a mobile digital reference service system that is compatible with all operating systems or platforms, and that can provide a fast, convenient, and reliable electronic service for users. The system supports Android and iOS mobile operating systems, as well as the web service of PC. The system can provide stable and reliable reference service to meet the demand from users (primarily the students and teachers), and can make its service compatible with a cross-platform environment, allowing it to access reference service at any time and place. Therefore, the objectives of the system are:

a) Based upon real situations and a user survey on user requirements (for example, a survey of university libraries), the system adopts the hybrid cross-platform technique to provide professional reference service to the users.

b) To set suitable reference service scenarios for users to efficiently accomplish operations, including Q\&A, comments, inquiry, and interaction on $\mathrm{PC}$ or mobile terminals.

c) The system is able to realize virtual service and real reference service, and can provide combined services to the users.

d) Due to the limitations on real operations, such as user interface of webpages and mobile terminals, the size of display, system performance, and the system graphic interface should be clear, simple, easy to interact with, and easy to manipulate.

To summarize, the reference service system that is based upon the hybrid frameworks will fully leverage the infrastructure, facilities, software, hardware, and local area web of local university libraries to realize the virtualized operation environment. It will ensure that the reference service system will run smoothly and interact conveniently with both the intranet and internet. As per the rules and regulations of local university libraries, the survey results on user requirements, and the comprehensive analysis of multiple factors (such as mobile network and cloud storage technology), the reference service system designed in the hybrid frameworks will ensure the security, high efficiency, and extensibility of the system data.

\subsection{The Overall Structure of Reference Service System (RSS)}

The overall structure of RSS consists of the client (mobile terminals and PC) and server. The client is responsible for displaying the human machine interface and realizing some basic functions, while the server is responsible for providing support on infrastructure, data, and standard service, and for dealing with various service requests from the client. The client side of RSS in the hybrid frameworks is composed of the application layer, the bridge layer, the assembly layer, and the device layer, as shown in Fig. 3.

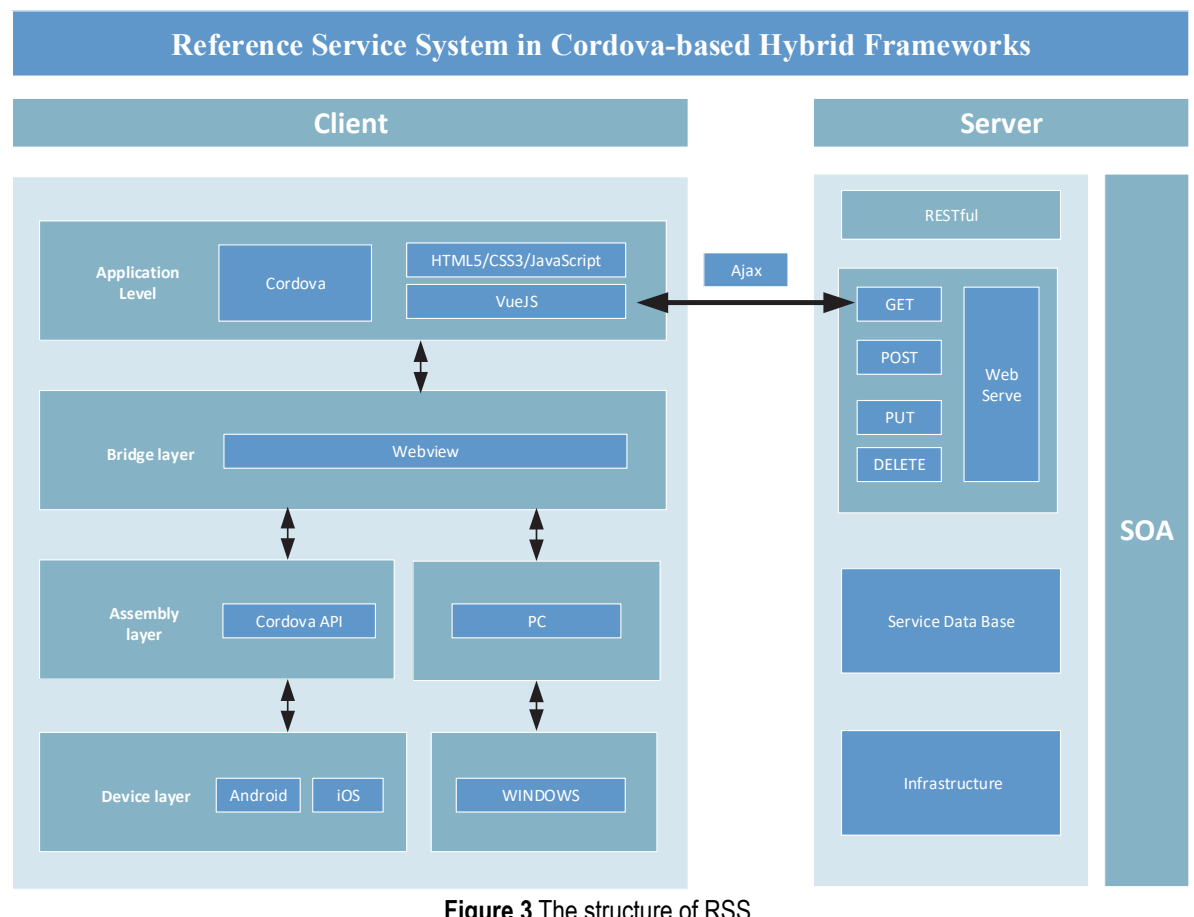

a) Application layer: it is responsible for displaying human machine interface, realizing business function, and communicating with the server. It uses Ionic framework to render the appearance and styles of applications, and the
Ajax technique to communicate with the server. It updates the business data with the assistance of HTML5, CSS3, and JavaScript. It also calls API of Cordova to realize the communication with devices at a low level. 
b) Bridge layer: it is the key for cross-platform operations, and is responsible for two-way communications between the original source code and JavaScript code. Cordova will package the way of bridging, which is dependent on the features of WebView of different platforms, and provides universal API interface for the front application layer.

c) Assembly layer: Cordova packages the interfaces/ports of different platforms and provides plugins for the bridge layer to call. It is compatible with an ordinary PC port.

d) Device layer: it covers the iOS and Android systems, and provides original API interface for the assembly layer to call. It also allows for the assembly layer to call in the Windows system.

\subsection{The Realization of RSS in Hybrid Frameworks}

When the client requests a question via a reference service, the question will, after clustering analysis, be classified into either conventional problems or nonconventional problems. If it is a simple and repeated conventional question, the server will call the database for conventional questions to seek the answer by comparing the key words contained within the question. If the question is complex and new, it will exchange with the professional consultants to seek the answer to the question via the realtime reference platform. After the system runs for a certain amount of time (based upon process tracking, the reference record, and the frequency of raised questions), it will, on the one hand, automatically add the frequently raised questions to the conventional question database to extend and improve the database. On the other hand, it will complement the conventional question database by requesting that the professional consultants check and screen these questions. The key procedures to classify and treat these questions are shown in Fig. 4.

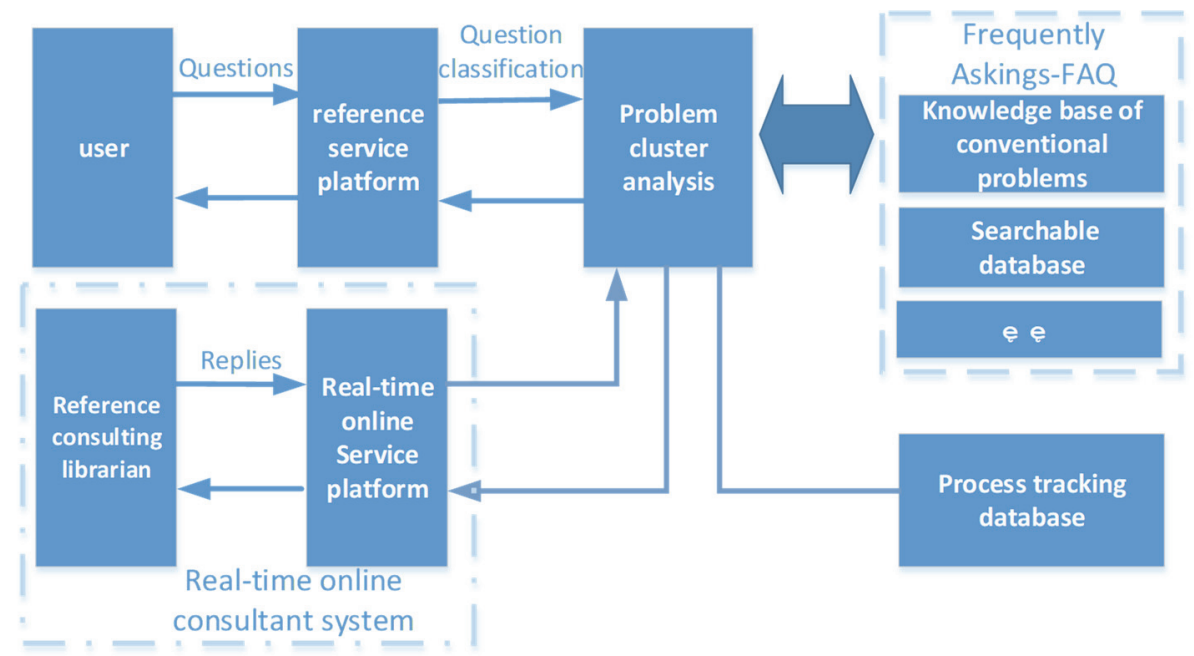

Figure 4 Main procedures to classify and treat references

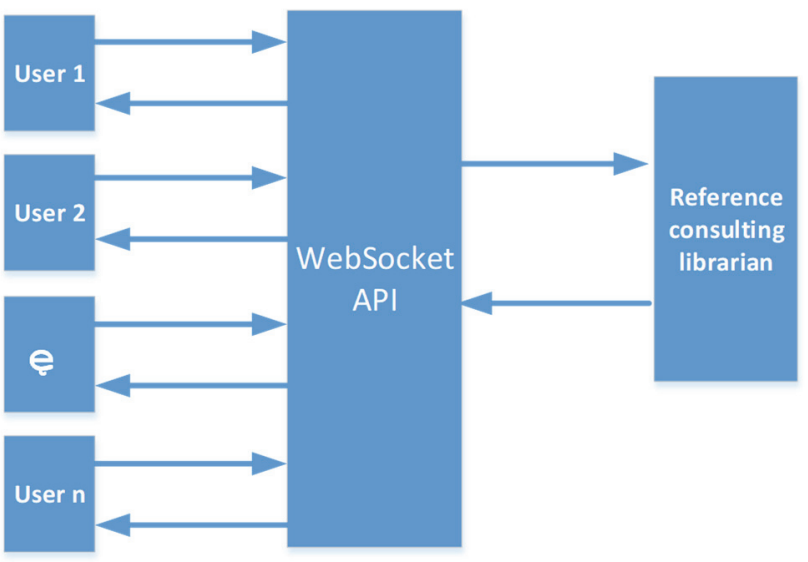

Figure 5 Establishing the online reference platform

The system adopts the WebSocket technique to realize duplex communication on a single TCP connection, and to establish an independent online reference platform. It also integrates the module into the whole development process in a compatible way, as shown in Fig. 5.

The successful implementation of RSS primarily relies upon the front-end and back-end development, which largely includes the following: a) Using the front-end development tool, WebStorm, it completes the design of webpages and partial front-end coding/programming, as shown in Fig. 6 and Fig. 7.

b) Using a Java-based, back-end development environment, IntelliJ IDEA, it completes the design and coding/programming of the back-stage control of the user login page. Part of the back-end coding/programming is shown in Fig. 8 and Fig. 9.

The data storage function of RSS primarily includes two parts: local storage and cloud storage on the network. The local cache data package is composed of structured and unstructured data. The former mostly involves user login information, relative reference record, and viewing history, which are all stored and managed by the built-in local database SQLite of HTML5. The latter is mainly stored in Elastic Compute Service (ECS) for management.

This convenient way of storing and sharing data online ensures the stability of the system and the quality of mobile service. The system response database has been successfully developed by building upon the relative technique of the distributed file storage database, MongoDB 3.2. The principal database sheet structure of the database is shown in Fig. 10. 


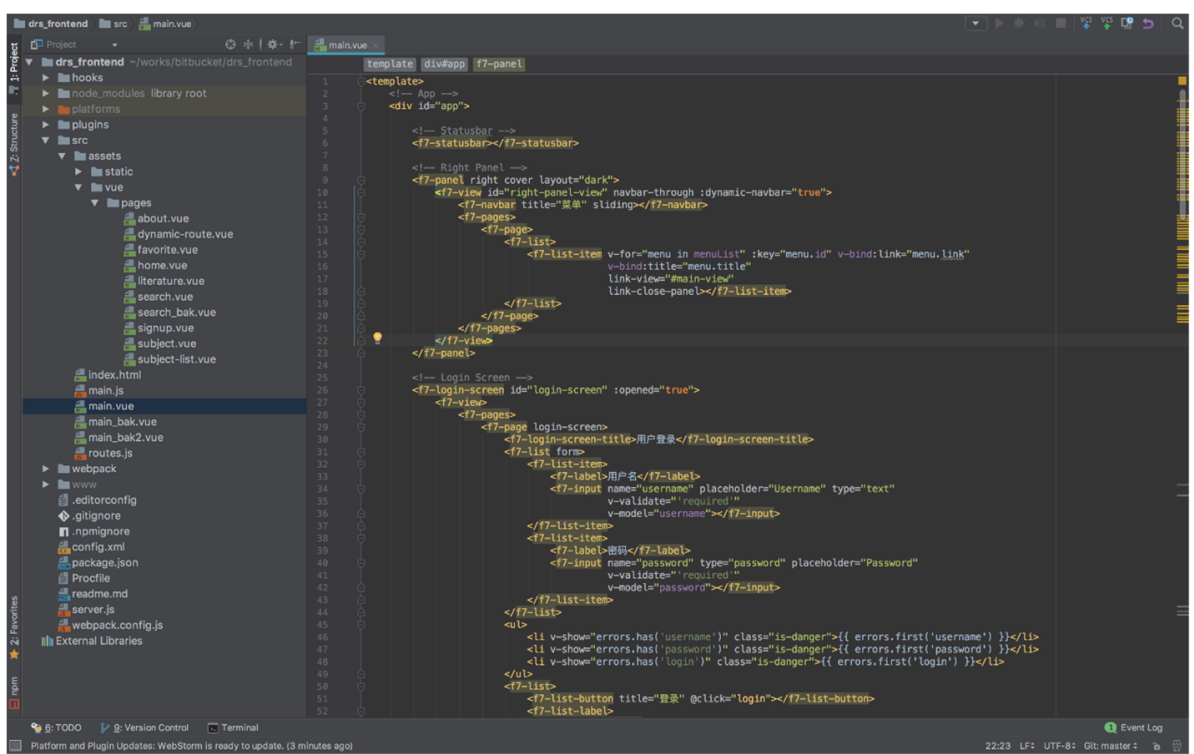

Figure 6 Coding of the system's homepage

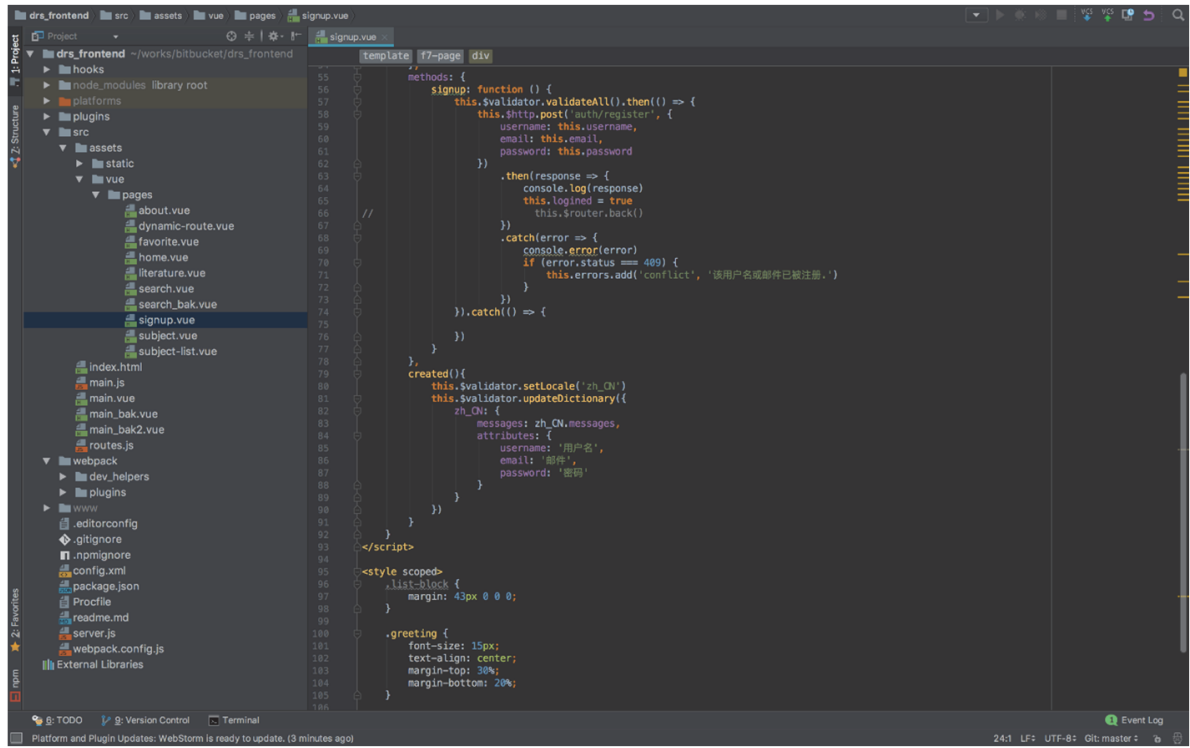

Figure 7 The design of the user register page

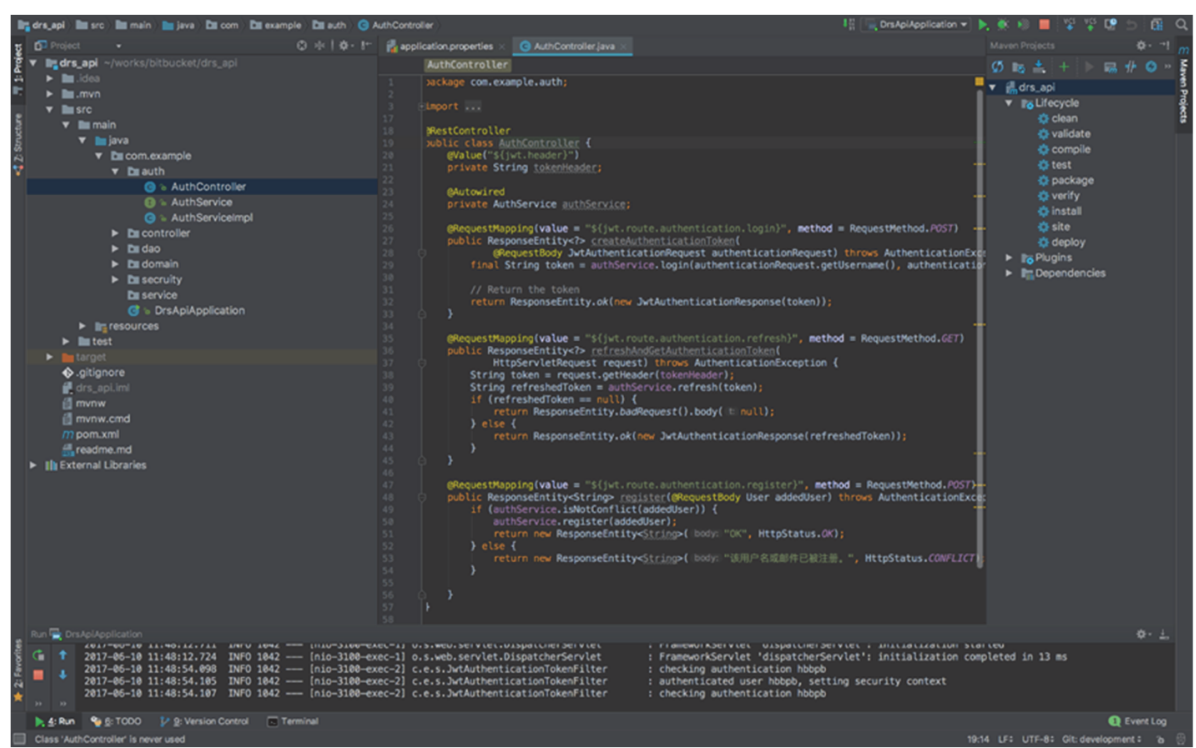

Figure 8 Login for system user controller 


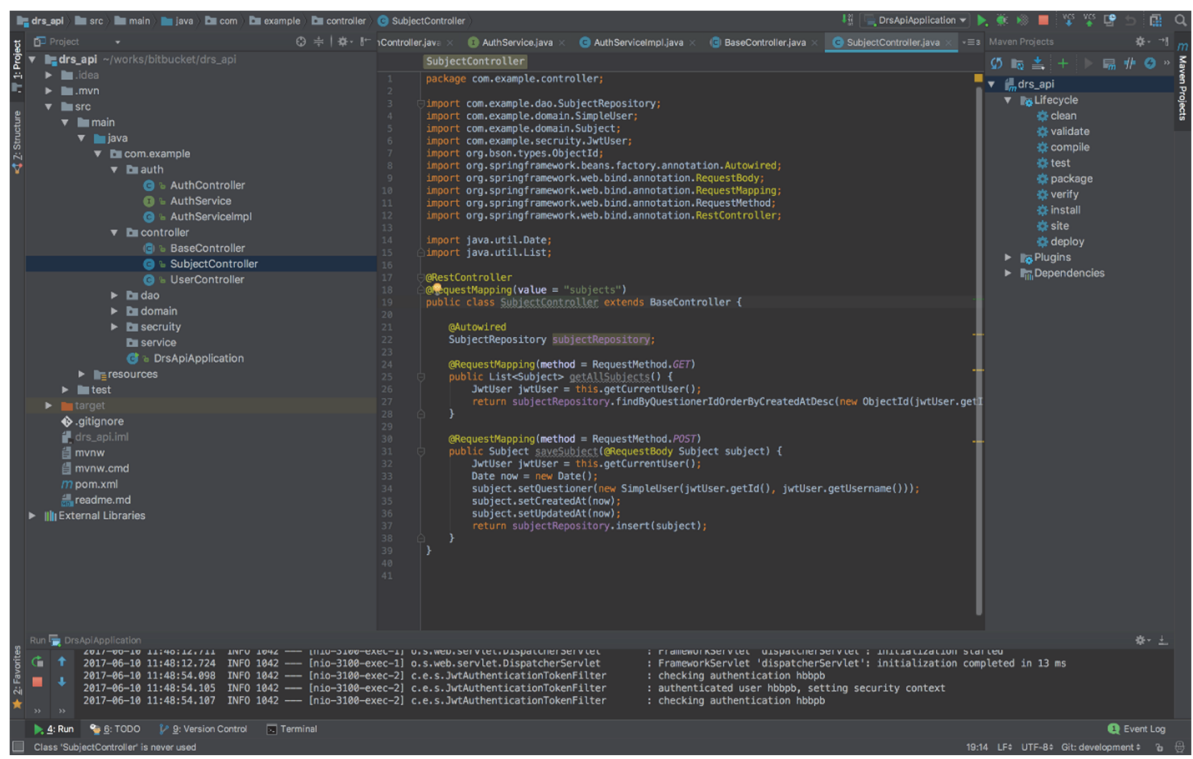

Figure 9 Raising a question for system user controller

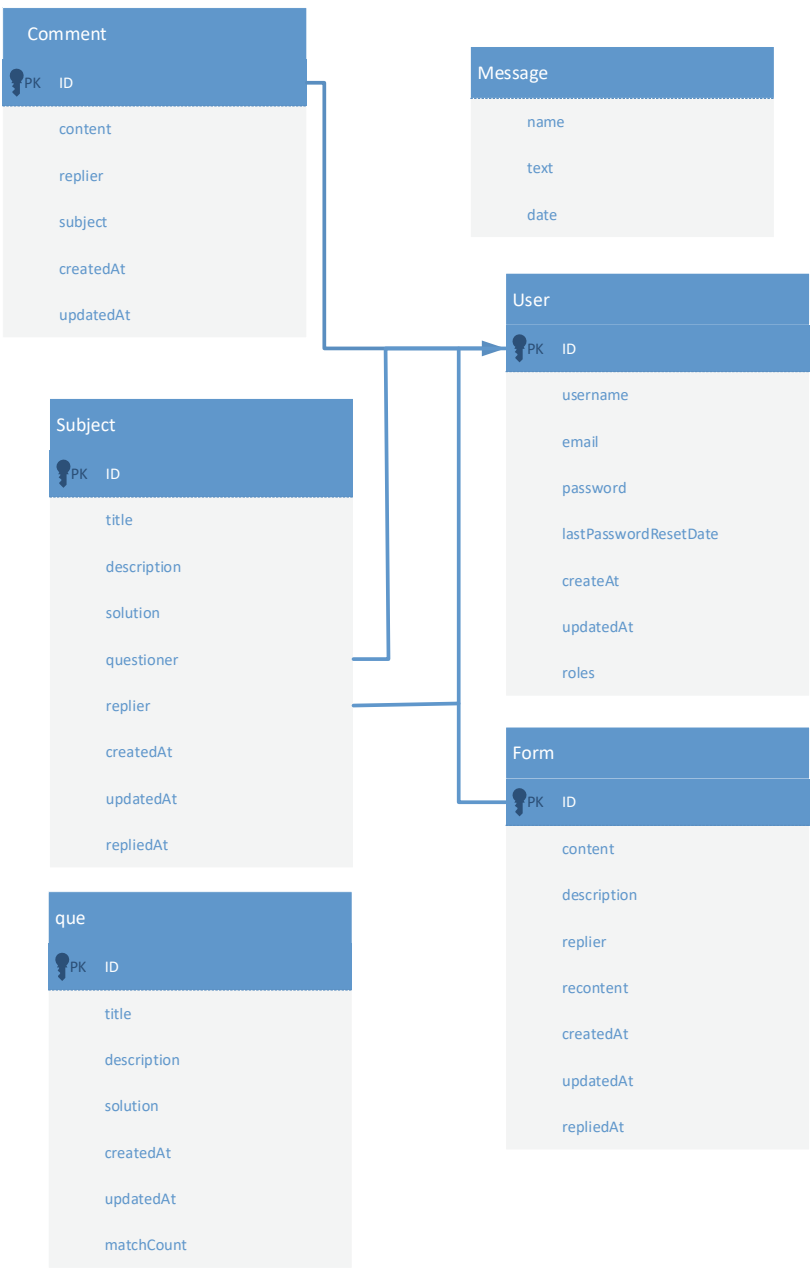

Figure 10 The structure of the database sheet.

\section{RUN RESULTS}

The run results of the real functions of RSS are largely3 reflected in the following facets:

a) The functions such as interface, register, query, and question are realized, as shown in Fig. 11.
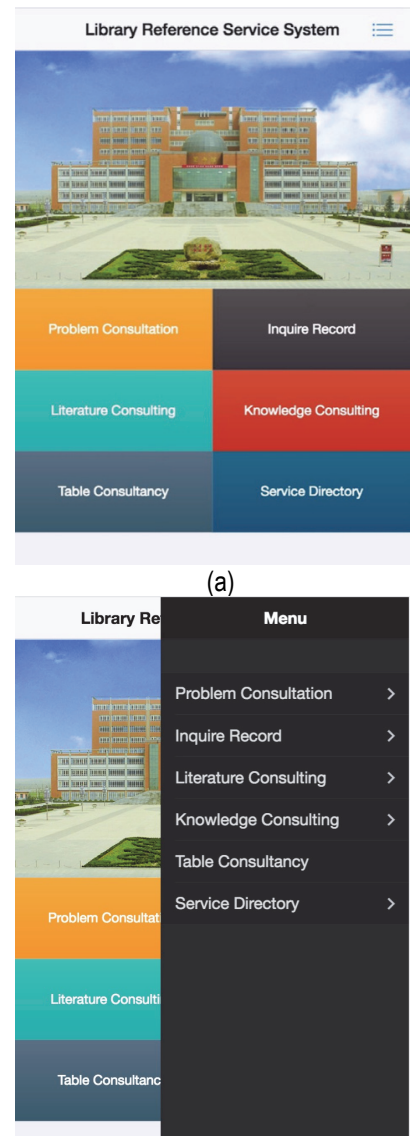

(c)

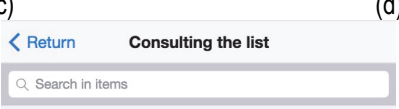

$\begin{array}{lr}\text { < Return } & \text { Problem Consultation } \\ \text { Title } & \text { Input the title in here }\end{array}$

Problem Descri..

Input the description in here.

(b)

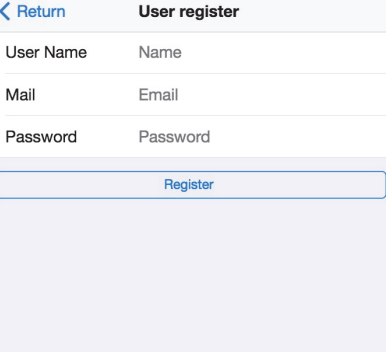

\section{title test}

have a problem

by hbbpb at 2019-03-09 22:49

$$
\begin{aligned}
& \text { test34 } \\
& \text { test345 } \\
& \text { by hbbpb at 2017-06-26 16:24 } \\
& \text { test2 } \\
& \text { test2 } \\
& \text { by hbbpb at 2017-06-25 18:12 }
\end{aligned}
$$

(e)

Figure 11 The interface of RSS: (a) system interface, (b) user register, (c) function menu, (d) Q\&A reference, (e) query information 
b) Users on PC can directly click open the service webpage, while mobile terminal users have to download installation package (drs_android. apk or drs_ios. apk) of the application to run the program and complete the installation of the application. The run results are shown in Fig. 12 and Fig. 13.

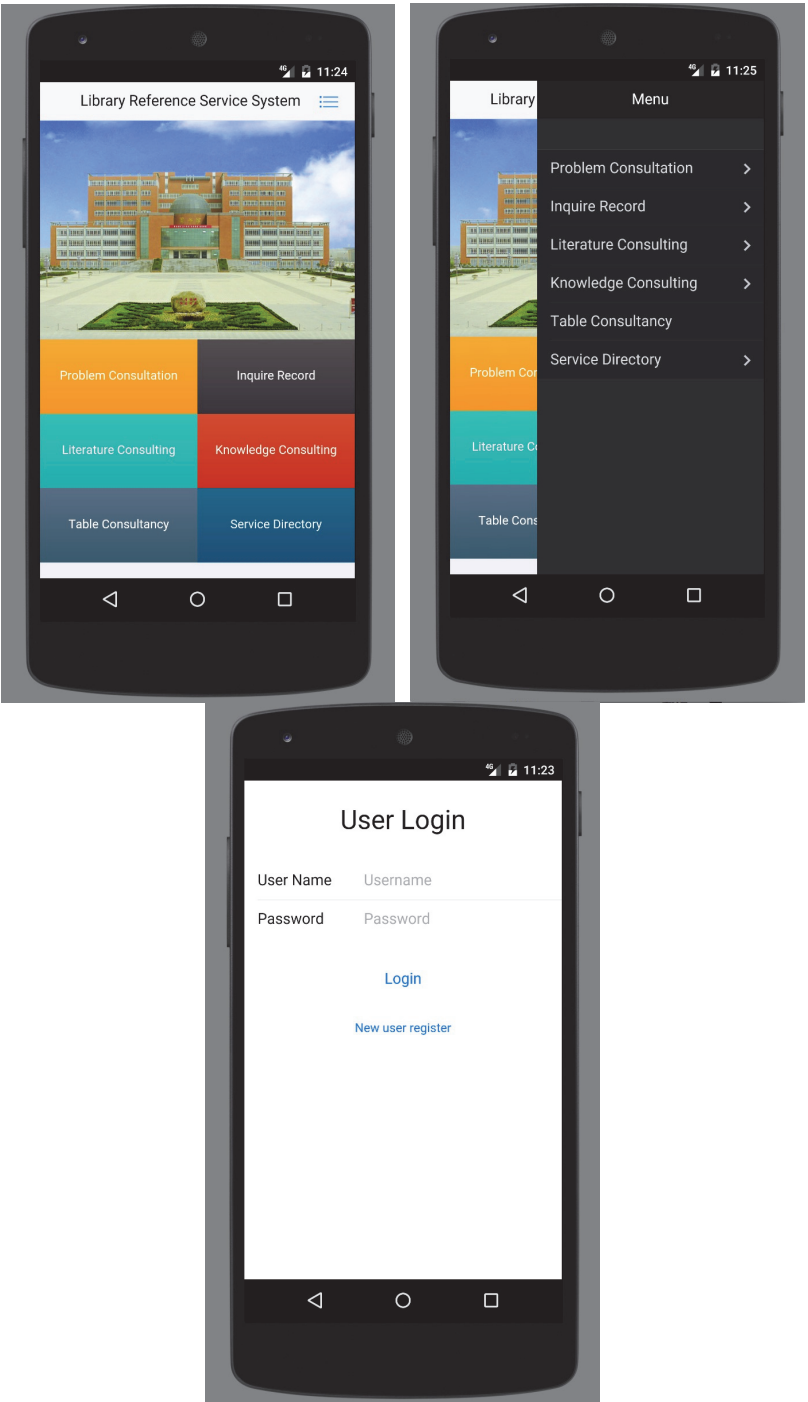

Figure 12 The login interface for mobile phones (APP)

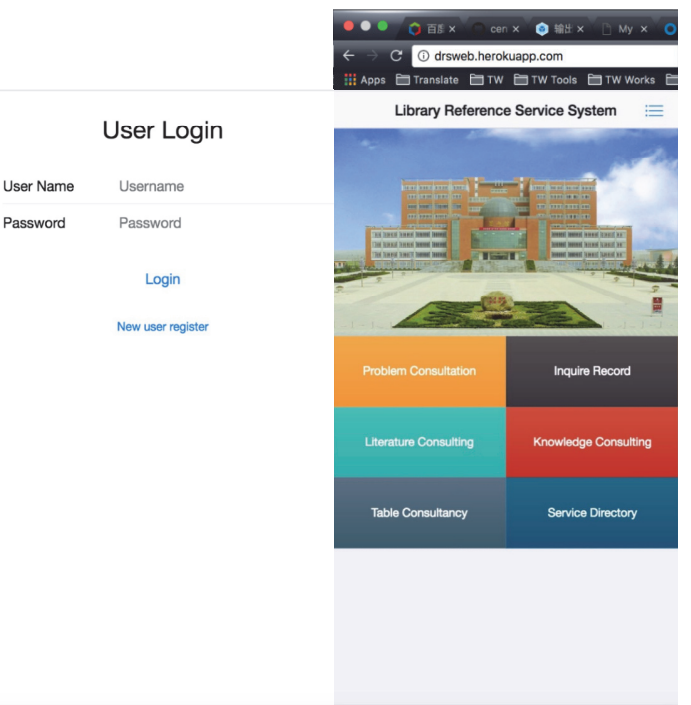

Figure 13 The login interface for webpage c) It finally completes the test on the interactive operating system in real time, debugs the functions of all the modules, and updates the database. The real-time communication test results are shown in Fig. 14.

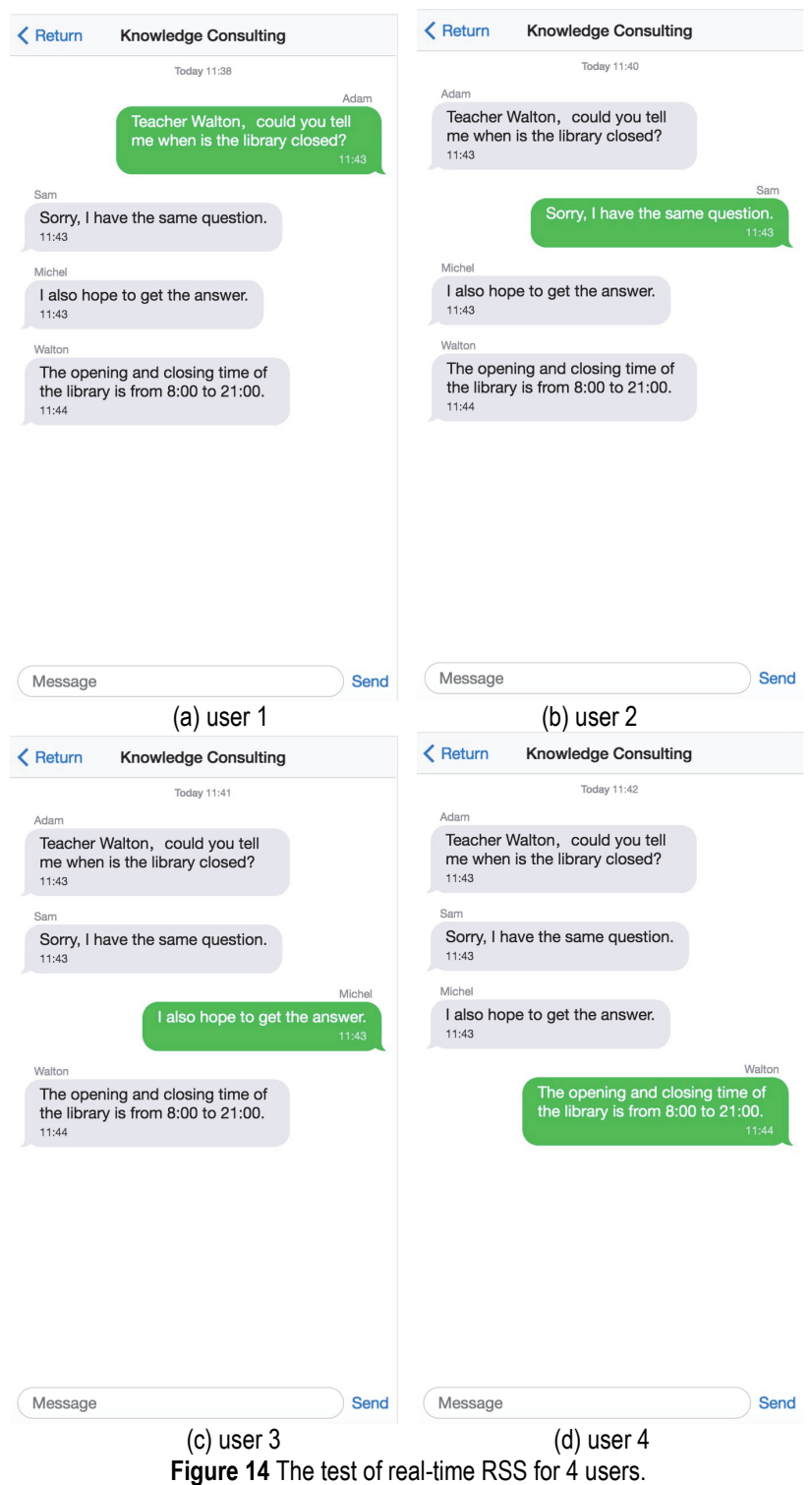

The Reference Consulting Service System studied in the paper is based upon the hybrid integration platform. After adopting the following main steps and repeated adjustments, it achieves the module functions the system mostly needs. The system uses Alibaba Cloud's ECS server to complete the server's resource configuration as per its minimum requirements. Besides, it also builds a local physical server. With WebStorm the development tool of the open sourced JavaScript, it realizes the front-end development of the system. With IntelliJ IDEA the integrated environment that is based upon Java language, it realizes the back-end development of the system. Basing upon the distributed file storage database MongoDB 3.2, it realizes the database development of the system. Users can directly open the webpage via a PC, or install the client portal by downloading the application package (drs android. apk or drs_ ios. ipa) via a mobile terminal, or directly run the program via the Web of UI. After carrying out the test and check of the real-time operating system, it 
basically completes the debugging work of the module functions.

The test results show that the designed system basically achieves the expectation, which is reflected in the following main aspects:

a) The system has good compatibility with the web system of mobile (Android and iOs) portal and PC.

b) The system can provide robust real-time online consulting service for multiple persons.

c) The learning FAQ database can collect consulting questions by consulting frequency and provide users with queries.

d) The functions of the system using a single or multiple ECS clouding data servers are stable.

In short, the system designed in this paper has been tested and verified, and all its functions have basically achieved the design objectives.

\section{CONCLUSION}

With the rapid development of communication technology, especially the booming development of computer network and mobile network technology, the users that require reference consulting service have changed, it is even more so for their needs and requirements. These changes will inevitably cause a chain reaction corresponding to the library reference consulting service business. At the same time, the booming of science and technology further promotes the development of reference consulting service. Accordingly, the main technology it depends upon will also be replaced inevitably, or improved by integrating multiple technology. New types of users and new business models will emerge while the traditional users and business models will coexist in a certain period. In response, a corresponding reference consulting service system will meet the needs of this period. The hybrid integration platform-based reference consulting service system is such a product that meets the needs of the current alternate phase. It makes full use of the booming telecommunication network and mobile network technology, and actively responds to the current urgent needs of users. The reference consulting service system that is based on the hybrid integration platform technology itself is a product of the digital and networked information era. The research and development of the hybrid integration platform based on reference consulting service system follows the future development trend of reference consulting service.

In response to the main problems regarding the buildup of mobile reference consulting service platforms for the libraries in China as mentioned in the preface, the paper develops a hybrid platform reference system that is based upon mobile terminals. By adopting Cordova hybrid platform and Web technology, it well fixes the incompatibility between PC terminal and mobile terminals, and between the two main mobile operating systems (iOs system and Android system), thereby creating an integrated hybrid platform system to some extent. The system enables users to fully take advantages of both the mature digital reference service and the emerging mobile reference service, thereby further extending the services in depth and breadth. At the same time, by adopting the ECS cloud server technology, it significantly cuts the physical cost on building the system and optimizes allocation of existing resources. The study will lower the budget constraint for the promotion and localization of the digital reference consulting services and mobile information services, and will help further promote and localize mobile reference consulting services, thus paving a new and cheap way to "popularize" reference consulting services. In this way, the system meets the needs of local universities in China by avoiding waste of resources and repetitiveness in building libraries. In a sense, it is conducive to the localization and popularization of the advanced reference services.

The hybrid integration platform-based reference consulting service system in the paper has some shortcomings as the system is currently only compatible with the two most commonly used mobile operating systems (iOs system and Android system). Therefore, it still needs to be further upgraded in terms of compatibility to adapt to other mobile operating systems. With the realization of the query function, feedback function and high-frequency problem entry function of the problem database, it will significantly reduce the amount of data entry work if further combining with artificial intelligence technology, and better provide users with convenient and quick query and feedback service. The abovementioned work will be further improved in subsequent research and development.

\section{Acknowledgements}

This project is supported by Key Project of Baoji University of Arts and Sciences (Grant YK2018012 and Grant BJWG201902), Scientific Research Project of Funded by Shaanxi Provincial Education Department (No. 20JK0483)

\section{REFERENCES}

[1] Lapidus, M., Bond, I., Wentz, E., et al. (2020). Measuring the quality of reference services provided by paraprofessionals at an academic library. The Journal of Academic Librarianship, 46(5), 102198. https://doi.org/10.1016/j.acalib.2020.102198

[2] Ocran, T. K., Underwood, E. P. G., \& Arthur, P. A. (2020). Strategies for successful implementation of mobile phone library services. The Journal of Academic Librarianship, 46(5), 102174. https://doi.org/10.1016/j.acalib.2020.102174

[3] Xie, J. \& Sun, L. (2015). Exploring Chinese students' perspective on reference services at Chinese academic libraries: A case study approach. Journal of Academic Librarianship, 41, 228-235. https://doi.org/10.1016/j.acalib.2015.04.002

[4] Li, B., Song, Y., Lu, X., \& Zhou, L. (2020). Making the digital turn: Identifying the user requirements of digital scholarship services in university libraries. The Journal of Academic Librarianship, 46(5), 102135. https://doi.org/10.1016/j.acalib.2020.102135

[5] Bertot, J., Estevez, E., \& Janowski, T. (2016). Universal and contextualized public services: Digital public service innovation framework. Government Information Quarterly, 33, 211-222. https://doi.org/10.1016/j.giq.2016.05.004

[6] Cathy, W. \& David, C. M. (2020). Faculty perceptions of librarians and library services: Exploring the impact of librarian faculty status and beyond. The Journal of Academic Librarianship, 46(5), 102200. 
https://doi.org/10.1016/j.acalib.2020.102200

[7] Wang, C. H., Huang, R. H., Li, J. Y., et al. (2020). Towards better information services: A framework for immigrant information needs and library services. Library \& Information Science Research, 42,101000. https://doi.org/10.1016/j.lisr.2019.101000

[8] Tara, M. (2020). User preferences related to virtual reference services in an academic library. The Journal of Academic Librarianship, 46(5), 102094. https://doi.org/10.1016/j.acalib.2019.102094

[9] Ali, E. E., Teo, A. K. S., Goh, S. X. L. et al. (2018). MedAdAppQ: A quality assessment tool for medication adherence apps on iOS and android platforms. Research in Social and Administrative Pharmacy 14, 1125-1133. https://doi.org/10.1016/j.sapharm.2018.01.006

[10] Knox, S., Moghadam, S., Patrick, K., Phan, A., \& Choo, K. K. R. (2020). What's really' Happning'? A forensic analysis of Android and iOS Happn dating apps. Computers \& Security 94, 101833. https://doi.org/10.1016/j.cose.2020.101833

[11] Chen, H. X. (2018): Evaluation of Personalized Service Level for Library Information Management Based on Fuzzy Analytic Hierarchy Process. Procedia Computer Science 131, 952-958. https://doi.org/10.1016/j.procs.2018.04.233

[12] Bosnic, S., Papp, I., \& Novak, S. (2017). The development of hybrid mobile applications with apache cordova, Telecommunications Forum, 1-4. https://doi.org/10.1109/TELFOR.2016.7818919

[13] Yang, Y., Zhang, Y., Xia, P., Li, B., \& Ren, Z. (2017). Mobile terminal development plan of cross-platform mobile application service platform based on ionic and cordova. International Conference on Industrial Informatics Computing Technology, Intelligent Technology, Industrial Information Integration, 100-103. https://doi.org/10.1109//CIICII.2017.28

[14] Liu, F., Xiao, M., \& Feng, W. (2015). Design of cordova based message push module for cross-platform smart home application. Fifth International Conference on Instrumentation and Measurement, Computer, Communication and Control, 635-639. https://doi.org/10.1109/IMCCC.2015.139

[15] Kudo, N., Yamauchi, T., \& Austin, T. H. (2017). Access control for plugins in Cordova-based hybrid applications. IEEE International Conference on Advanced Information NETWORKI NG and Applications, 1063-1069. https://doi.org/10.1109/AINA.2017.61

\section{Contact information:}

\section{Dengke XIN}

School of Electrical and Control Engineering,

Shaanxi University of Science \& Technology,

Xi'an 710021, China

E-mail:757815429@qq.com

\section{Lei ZHANG}

(Corresponding author)

School of Physics and Optoelectronic Technology,

Baoji University of Arts and Sciences,

Weibin District, Baoji 721016, China

E-mail: simpleman2003@163.com

\section{Xiaoliang YANG}

University Library Department,

Baoji University of Arts and Sciences,

Weibin District, Baoji 721016, China

E-mail: aliang862_xtu@126.com

\section{Yuan FANG}

School of Electrical \& Electronic Engineering,

Baoji University of Arts and Sciences,

Weibin District, Baoji 721016, China

E-mail: fangyuanmy@163.com 